\title{
Predictors of treatment failure and clinical stability in patients with community acquired pneumonia
}

\author{
Deirdre Morley ${ }^{1}$, Antoni Torres ${ }^{2,3}$, Catia Cillóniz ${ }^{2,3}$, Ignacio Martin-Loeches ${ }^{1,4}$ \\ ${ }^{1}$ Multidisciplinary Intensive Care Research Organization (MICRO), St James's University Hospital, Dublin, Ireland; ${ }^{2}$ Department of Pneumology, \\ Hospital Clinic Barcelona, Universidad Barcelona, Barcelona, Spain; ${ }^{3}$ Centro de Investigación Biomédica En Red de Enfermedades Respiratorias \\ (CIBERES), Madrid, Spain; ${ }^{4}$ Department of Clinical Medicine, Trinity Centre for Health Sciences, Dublin, Ireland \\ Contributions: (I) Conception and design: All authors; (II) Administrative support: All authors; (III) Provision of study materials or patients: All \\ authors; (IV) Collection and assembly of data: All authors; (V) Data analysis and interpretation: All authors; (VI) Manuscript writing: All authors; (VII) \\ Final approval of manuscript: All authors. \\ Correspondence to: Ignacio Martin-Loeches. Multidisciplinary Intensive Care Research Organization (MICRO), Wellcome Trust-HRB \\ Clinical Research, Department of Clinical Medicine, Trinity Centre for Health Sciences, St James's University Hospital, Dublin, Ireland. \\ Email: drmartinloeches@gmail.com.
}

\begin{abstract}
Community acquired pneumonia (CAP) is the leading infectious cause of mortality worldwide with approximately $10 \%$ of patients hospitalized requiring intensive care unit (ICU) admission. The ability to predict clinical stability (CS) and treatment failure (TF) enables the clinician to alter antibiotics appropriately, facilitate a timely ICU admission, or arrange a suitable discharge. The detection of CS and TF can be difficult and changes in clinical signs may be subtle or delayed. Thus clinical scores and biomarkers are routinely used to identify severity and monitor clinical progression. The evidence, however, is vast and the definitive role of these systems is at times difficult to elucidate. The aim of this review is to analyse the current literature and to provide a rational and clinically focused view of the predictive utility of various systems used to identify CS and TF in CAP.
\end{abstract}

Keywords: Pneumonia; community acquired pneumonia (CAP); sepsis; treatment failure (TF); biomarkers; procalcitonin (PCT)

Submitted Apr 24, 2017. Accepted for publication Jun 03, 2017.

doi: $10.21037 /$ atm.2017.06.54

View this article at: http://dx.doi.org/10.21037/atm.2017.06.54

\section{Introduction}

Pneumonia is the leading infectious cause of mortality worldwide (1). Community acquired pneumonia (CAP) is associated with a high mortality especially among the elderly and those with premorbid conditions (2,3). Approximately $10 \%$ of patients hospitalized require intensive care unit (ICU) admission $(4,5)$. In addition there are a significant proportion of patients discharged from hospital that are readmitted within 30 days (6).

Early identification of severe disease and careful monitoring of treatment response is essential when managing patients. For this reason the concepts of clinical stability (CS) and treatment failure (TF) have been extensively studied. It follows that much research is focused on identifying useful surrogates for CS and TF. The evidence, however, is vast and the definitive role of biomarkers or clinical scores is at times difficult to elucidate. The aim of this review is to analyse the current literature and to provide a rational and clinically focused view of the predictive utility of various systems used to identify CS and TF in CAP.

\section{Current concepts of CS and TF}

CS and TF are essential components of patient care. The ability to predict CS and TF enables the clinician to alter antibiotics appropriately imperative in the era of antibiotic resistance and antibiotic related complications. It also 
facilitates a timely ICU admission and escalation in care, or alternatively an early appropriate discharge. A longer time to CS, or persistent TF is significantly associated with an excessive inflammatory response, poor clinical outcomes, a higher rate of pneumonia related complications, and prolonged length of stay and ICU admission (7-10). In addition, time to CS and presence of TF are considered crucial endpoints in the literature, similar to mortality outcomes (10-12).

In an effort to standardize clinical endpoints in the literature and create useful clinical tools a number of definitions for CS exist. These include the American Thoracic Society and Infectious Disease Society of America (ATS 2001 and ATS/IDSA 2007) and Halms criteria $(13,14)$. Using these criteria it has been demonstrated that the median time to CS in patients with CAP is between 2-4 days $(11,13)$. Interestingly, another important consideration is the actual sequence of clinical symptom and sign resolution. This varies greatly between patients and specific resolution patterns maybe associated with worse outcomes (15). In practice if CS is not achieved within 3 days, further investigation, treatment escalation or even ICU admission may be warranted.

Conversely TF refers to ongoing infectious symptoms and signs while receiving treatment (4). Mortality increases several folds if TF is present (8). In the presence of TF within the first 3 days care providers should be concerned about a progression of primary pneumonia, and the need for treatment escalation or even ICU admission. Late failure may indicate pneumonia related complications or a super imposed infection. This differs from non-responding pneumonia in which there may not be a clinical deterioration (16). TF is subdivided in to early (within 72 hours of hospital admission) or late and can occur in up to $31 \%$ or $11 \%$ of inpatient admissions respectively (17).

\section{Systems to identify disease severity and monitor clinical progression}

The detection of CS and TF can be difficult and changes in clinical signs may be subtle or delayed (4). Various clinical scores have been developed.

Mortality scores include the Pneumonia Severity Index (PSI) and CURB-65 are validated for single use at the time of initial assessment. They are primarily designed to identify low risk patients suitable for outpatient management and are therefor less effective at identifying patients with severe disease $(18,19)$.
The PSI boasts better external validity when compared to the CURB 65. However, it consists of 20 variables and is therefor complicated to use in clinical practice. In addition it has not been validated for use on certain patient groups for example immunosuppressed patients and pregnant women $(4,20)$. The CURB-65 (or simpler CRB-65) is easier to use consisting of just five parameters (confusion defined as mental test score $\leq 8$, uraemia $>7 \mathrm{mmol} / \mathrm{L}$, respiratory rate $\geq 30$ breaths/minute, blood pressure systolic $<90 \mathrm{mmHg}$ or diastolic $\leq 60 \mathrm{mmHg}$, age of 65 years or more). CURB-65 has not been as extensively validated as the PSI. In addition, similar to the PSI, the CURB-65 score is not recommended for use in the immunosuppressed population (4). Both scores, in particular the CURB65, may underestimate mortality in the lower scoring patients (21). Both scores lack specificity when identifying patient with severe disease who require admission to ICU (19). The IDSA and ATS have issued revised based on the PSI and CURB-65 score for those with severe disease (4). IDSA/ ATS minor criteria has a high negative predictive value in the prediction of "emergency" CAP (patients that will require mechanical ventilation, vasopressors or who have a high risk of death) (22). However, CURB-65 has been shown to be superior to IDSA/ATS minor criteria in the prediction of mortality in patients with severe disease in a low mortality setting (hospital mortality 1.3\%) (23).

CS scores also exist most notably the Halms and ATS criteria. The advantage of these criteria is that they can be used to evaluate treatment response over time where normalization of various parameters equates to the achievement of CS $(4,13)$. Both perform well, and strongly correlate to mortality and risk of complications. For example, in a large prospective observational study Akram et al. demonstrated an area under receiver operating characteristic curve (AUC) for 30-day mortality of 0.95 and 0.94 for Halms and ATS respectively (24). Although Halms performs slightly better, ATS is easier to use with 4 variables compared to the 7 components of the Halms. Both are widely used as end-points for CS in clinical trials $(8,24,25)$.

In summary, while all scores correlate well to mortality and risk of complications, simple and easier to use criteria are more likely to be applied in clinical practice. In addition, biomarker surrogates of host immune response are not included in these scores although it has been demonstrated that immune dysregulation correlates with a poorer prognosis (26). 
Table 1 Characteristics of CRP

Secretion (hours from exposure)
Starts: $4-6$
Peaks: 36
Factors that affect concentration levels
Increase
Inflammatory disease: rheumatoid arthritis, psoriatic arthritis,
vasculitis, Crohns disease
Trauma: surgery, burns, fracture
Myocardial Infarction
Pancreatitis
Fungal infection
Malignancy: lymphoma, leukaemia
Decrease
Early infection
Liver injury
Drugs: corticosteroid

CRP, C-reactive protein.

\section{Biomarkers}

\section{Biomarkers as surrogates of disease severity}

Biomarkers are seen as an effective way of monitoring host response to infection. They are widely used to monitor disease severity and response to treatment in CAP. The search for the ideal molecular surrogate is ongoing. Although multiple biomarkers are undergoing rigorous investigation, C-reactive protein (CRP) and procalcitonin (PCT) remain the most robust and widely used $(27,28)$. Among the pro-inflammatory cytokines, interleukin 6 (IL-6) has been of particular interest in the literature $(29,30)$.

CRP, PCT and IL-6 differ in the timing of response to a stimulus and half-life (Tables 1-3). CRP is predominantly synthesized in the liver in response to IL-6 induction of CRP mRNA $(27,31)$. The single determinant of CRP level is the rate of synthesis in the liver in response to the inflammation intensity $(27,32)$. In this way it is superior to more complex acute phase characteristics such as leukocytosis and fever (27). PCT is a 116 amino acid prohormone of calcitonin (31). In the event of a bacterial infection the CALC-1 gene is up regulated producing PCT in large amounts by macrophage and monocytic cells throughout the body especially the liver, lung and intestine (33). Some
Table 2 Characteristics of PCT

Secretion (hours from exposure)
Starts: $2-3$
Peaks: 6
Factors that affect concentration levels
Increase
Inflammatory disease
Trauma: surgery, burns, fracture
Myocardial infarction
Pancreatitis
Renal failure
Acute respiratory distress syndrome
Fungal infection
Drugs: antithymocyte globulin
Malignancy: medullary thyroid carcinoma, small-cell lung
carcinoma
Decrease
Early infection
Localised infection
Subacute endocarditis

PCT, procalcitonin.

studies have demonstrated that it is a more sensitive and specific diagnostic marker of sepsis and CAP than CRP $(34,35)$. The rise in PCT is more immediate when compared to CRP, due to cytokine like behavior. It is identified within 2-3 hours with a peak at 6 hours. The halflife is 24 hours (31). Conversely CRP secretion begins at 4-6 hours and peaks at 36-50 hours thereby potentially limiting its efficacy in predicting early TF $(27,36)$. One conceivable advantage of IL-6 over CRP and PCT is an immediate response to infection $(37,38)$. Another potential diagnostic advantage of IL-6 over PCT is that it is a more sensitive marker of localized infection, for example effusions (38) (Tables 1-3).

The role of biomarkers as a marker of severity and as a predictor of CS is well described $(25,39,40)$. In a sizable prospective cohort study of 394 patients admitted to two large Spanish hospitals with CAP, 55.8\% reached CS as defined by a modified version of the Halms criteria within 72 hours. As expected median levels of CRP, PCT, IL-6, IL-10 were significantly higher in patients 
Table 3 Characteristics of IL-6

Secretion (hours from exposure)
Starts: immediate
Peaks: $2-4$
Factors that affect concentration levels
Increase
Inflammatory disease: rheumatoid arthritis, Crohns disease
Lymphoproliferative disease: Castleman's disease
Malignancy: multiple myeloma, prostate carcinoma, ovarian
carcinoma
Cardiac myxoma
Decrease
Late infection

who did not reach CS. Interestingly, although a small number of patients were used in the multivariate analysis, Menendez et al. successfully demonstrated that CRP was a significant independent predictor for the absence of severe complications slightly more than PCT (25). A larger prospective study of 570 patients admitted via the emergency department and acute medical unit in a large Scottish hospital demonstrated that CRP $<100 \mathrm{mg} / \mathrm{L}$ on admission was significantly associated with reduced 30-day mortality (OR 0.18, P=0.03), need for mechanical ventilation and/or inotropic support (OR $0.21, \mathrm{P}=0.002)$ and complicated pneumonia (OR 0.05; $\mathrm{P}=0.003$ ) (39). Similar to the Menendez study the negative predictive value of CRP for excluding severe complications (30 day mortality) in this investigation compared favorably to severity scores, this time to the CURB-65 and PSI. Other studies have demonstrated that Il- 6 maybe a useful predictor of TF and mortality. In one hospitalized patients with elevated IL-6 were shown to have a higher risk of 30-day mortality with a sensitivity of $84 \%$ and specificity of $87 \%$. In this prospective trial of 101 patients $(24.8 \%$ mortality) there was good correlation between IL-6 levels and clinical severity scores (PSI, CURB 65 and MEWS). Interestingly, in this study comparator PCT levels did not reach significance (30).

\section{Signalling or functioning, are biomarkers good surrogates for clinical cure?}

Ideally biomarkers should be of high specificity and sensitivity, and useful for characterizing severity and monitoring response to treatment even in the absence of clinical signs $(31,41)$. It is well described that patients with severe CAP who mount an excessive systemic pro inflammatory response are more likely to have a deleterious outcome. This immune response is complex however and biomarker dynamics may not be specific to the underlying disease and clinical status of the patient (26). When monitoring biomarker levels confounding factors should be taken in to consideration for example the effect of age, antibiotic pretreatment, corticosteroids, chronic hepatic disease, renal impairment and viral confection (10,42-45). For example, there is concern that the PCT response in patients with influenza, particularly influenza A (H1N1) PDM 09 may relate to the severity of viral pneumonitis as oppose to the bacterial co-infection $(43,46)$. However, recently this was disputed when a study of 972 patients with H1N1 found that a cut off PCT of $<0.29 \mathrm{ng} / \mathrm{mL}$ in a population with a $20 \%$ prevalence of community acquired respiratory coinfection has a high negative predictive value of $92 \%$. PCT, therefor, appears to be effective at excluding co-infection in this group of patients (47). That said, it is important to interpret biomarker results cautiously and to correlate the results with clinical findings.

Biomarkers have a number of limitations (Tables 1-3). Criticism of the role of CRP in diagnostics surrounds the delay in response to a clinical stimulus and poor specificity. It is elevated in a variety of pathology for example trauma, surgery, burns, and immunological mediated inflammatory diseases. In addition, caution should be taken when interpreting CRP levels in patients with hepatic injury and following corticosteroid use $(48,49)$. PCT, in turn, is elevated in variety non-infectious conditions for example cirrhosis, pancreatitis, and mesenteric infarction, burns and aspiration pneumonitis $(38,50,51)$. Furthermore the diagnostic and predictive power of PCT declines in patients with severe sepsis, and in localized infection for example in endocarditis and empyema $(38,46,52)$. PCT levels should be interpreted with caution in impaired renal function (48). Studies differ as to appropriate negative cut-off point $(38,53)$. IL-6, a relatively novel biomarker, is involved in a variety of haematopoietic, immune and inflammatory responses. It enhances $\mathrm{T}$ cell differentiation through the induction of IL-2. It acts as a pyrogen in the presence of infection or inflammation $(54,55)$. Cytokine half-lives are short however and may decrease rapidly on clinical presentation. Cytokines also inherently lack specificity and are raised in a variety of inflammatory syndromes $(29,56)$. Therefor overexpression of IL-6 is 
associated with a range of conditions including multiple myeloma, Castlemans disease and rheumatoid arthritis (57). Validated cut-off points for IL-6 are not yet defined.

The prevailing finding seems to be is that higher levels of CRP and other biomarkers are present in patients who develop shock, severe respiratory failure, or who are at high risk of death reflective of the inflammatory host response to infection $(58,59)$. Menendez discovered that an initial CRP of $>25 \mathrm{mg} / \mathrm{dL}$ was associated double mortality risk compared with other patients with lower CRP with the same initial PSI stratification score (58). Outcomes are not always unanimous in this respect, however, for the reasons outlined above. For example, some studies report low CRP in severe sepsis, suggesting that CRP may have a semi-protective role (60). This is mainly reflected in animal studies however Que et al. described a low admission CRP in patients with severe streptococcal pneumonia that was associated with an increased risk of death. This was a modest retrospective analysis of 77 patients with streptococcal pneumonia of whom $12(15 \%)$ died. The results were significant $(\mathrm{P}=0.034)$, a $\mathrm{CRP}$ of $<169.5 \mathrm{mg} / \mathrm{L}$ predicted mortality in these patients with a sensitivity and specificity of $75 \%$ and $72 \%$ respectively (61).

Therefor it is important to appreciate that at times biomarker response is unpredictable. It is essential to interpret all results with caution and in the context of the changing clinical syndrome.

\section{Biomarker dynamics in severe disease and in treatment response}

Another noteworthy consideration is the subtleties of biomarker dynamics. A number of studies have demonstrated that the rate of CRP or PCT change correlates with response to treatment or, inversely, with risk of complications.

CRP kinetics is slower than that of PCT. A failure to decline after 3-5 days of antibiotic treatment is highly suggestive of TF, and highlights the need for antibiotic modification and further investigation for complications or other missed diagnosis. For example, Chalmers et al. demonstrated that failure of surveillance CRP to fall by $50 \%$ or more at day 4 is associated with increased risk of 30-day mortality ( $\mathrm{OR} 24.5 ; \mathrm{P}=0.001)$, need for mechanical ventilation $(\mathrm{OR} 7.1 ; \mathrm{P}=0.0001)$ and complicated pneumonia (OR 15.4, $\mathrm{P}=0.0001)$ (39). Similarly in another study the rate of CRP decline during the first 5 days of ICU admission in particular after day 2 was significantly associated with hospital mortality $(\mathrm{OR}=1.03, \mathrm{P}<0.001)$.
A patient with a daily decline in CRP of $10 \%$ per day has $32 \%$ reduced risk of mortality when compared with a similar patient with no CRP decrease (28). A multicenter study that focused on the role of consecutive CRP measurements in predicting TF discovered that a decline of CRP $<60 \%$ in 3 days or $<90 \%$ in 7 days was associated with inappropriate empiric antibiotic treatment (62). Coelho et al. considered CRP ratios at days 3, 5 and 7 in relation to day 1 CRP concentration during the first week of admission to ICU. A ratio that was higher than 0.5 at day 5 was associated with a fivefold increased risk of death in the ICU. Patients with no CRP decline over the first 7 days had significantly higher mortality (mortality of $43.2 \%$ compared to $9.5 \%$ in those with faster CRP response $\mathrm{P}=0.001$ ) (63). Interestingly Menendez discovered that CRP at day 1 had a high negative predictive value for early $\mathrm{TF}$ in addition to late failure predictive ability (59).

PCT conversely has more rapid dynamics and is useful therefor at identifying TF at an earlier stage (59). A study of 100 patients with CAP demonstrated that a PCT risk from day 1 to day 3 of ICU admission was independent risk factor for mortality $(\mathrm{OR}>4)$, while a day 3 PCT value of $<0.95 \mathrm{ng} / \mathrm{mL}$ was associated with a $95 \%$ survival probability (64). Similarly another observational cohort study of ICU patients with sepsis (52\% with pneumonia) determined that failure of PCT to decline by $30 \%$ between days 2 and 3 of treatment reflected the need for treatment modification (65). Martin-Loeches also discovered in a case control study (TF versus non TF patients) that PCT may be more valuable at discriminating failure than 1L-6 and CRP (66). A Danish study demonstrated that an increase in PCT is an early independent predictor of all cause mortality over 90 days follow up period and was superior to CRP and PCT (67).

\section{Biomarkers as a guide to treatment}

There has been a lot of interest in biomarker directed treatment strategies. Perhaps the most encouraging data surround the negative predictive value of PCT as a guide to antibiotic initiation and discontinuation. Multiple large studies have shown that following a PCT algorithm is associated with a reduction in antibiotic use in pneumonia without increasing risk of complications. PCT is effective in rationalizing antibiotics in patients with COPD and asthma requiring hospital admission $(68,69)$.

In particular the ProREAL study group, through an observational multinational multicenter prospective study 
demonstrated a successful reduction in antibiotic exposure from 7.4 to 5.9 days using $0.25 \mathrm{ng} / \mathrm{mL}$ as the recommended level for antibiotic consideration (70). Similar conclusions can be derived from a number of randomized controlled trials (71-74). The ProHOSP group also demonstrated a decline in antibiotic adverse effects with PCT guided antibiotic discontinuation (72).

The PRORATA trial considered critically ill patients with a range of bacterial infections (approx. 70\% pulmonary). Although PCT directed therapy reduced antibiotic exposure, there was no difference in length of ICU admission. In addition, there was a slightly higher mortality in the PCT group between days 29-60 although after correction for confounders this was not significantly different (75). A systematic review of PCT antibiotic algorithm RCTs found that there was no significant difference in mortality between PCT patients control patients (OR 0.91) (76). More recently, however, a large multicenter RCT (SAPS study) of critically ill patients with infection demonstrated a significant decrease in mortality at 28 days and 1 year when using PCT guided treatment compared to standard of care (74). Moreover, PCT may have a more powerful negative predictive value in identifying patients who need ICU admission when compared to CRP and cytokines including IL-6 (77).

\section{New discovery of molecules or paradigms}

\section{Novel biomarkers}

The evidence for use of biomarkers is vast and ever evolving. Most research surrounds the use of PCT and CRP. An elevated cytokine level, especially IL-6, correlates with disease severity and maybe more predictive of an early clinical deterioration than older biomarkers $(29,30)$. Other note worthy biomarkers include hormone surrogates [pro-adrenomedullin (ProADM), copeptin], markers of coagulation (D-dimer) among others (48,78-80).

Severe CAP is associated with a median cytokine concentration that has been shown to be twice as high as that of non-severe CAP (2). Zobel et al. also confirmed that elevated IL-6 was a strong predictor of 30-day mortality, with the median concentration of IL-6 significantly higher in patients who had a severe CAP course (54.4 vs. $16.6 \mathrm{pg} / \mathrm{mL}, \mathrm{P}<0.001)$ (29). Similarly Menendez et al. demonstrated in an earlier study that along with CRP, IL-6 was an independent predictor for 30-day mortality (58). Martin-Loeches et al. demonstrated that IL-6 was superior to PCT, CRP, IL-1 and IL8 in the prediction of early TF on day 1. IL-6 also had a high predictive value for late $\mathrm{TF}$ also (66). Interestingly studies suggest that genetic variability in the production of pro-inflammatory cytokines, in particular higher production of TNF alpha, IL-6, or the anti-inflammatory IL-10 is associated with higher mortality in sepsis including CAP $(81,82)$. A large ICU based observational study verified that an IL-6 174 GG genotype was associated with less ARDS, Septic shock and MODs disease in ICU patients with CAP (83).

ProADM and copeptin are stable surrogates of sepsis induced stress hormones. ProADM is a fragment of the vasodilator and bacteriocidal hormone adrenomedullin, particularly elevated in sepsis (84-86). Copeptin, a precursor of arginine vasopressin is raised in a variety of diseases including congestive heart failure but levels correlate significantly with poor outcomes in sepsis and CAP (80,87-89). Pro-ADM and Copeptin have been shown to be strong predictors of early mortality and adverse outcomes, potentially more than PCT and CRP. A systematic review of 12 studies confirms that an increased MR proADM is associated with increased risk of short term mortality and that a combination of proADM and CURB-65 is associated with an improved predictive efficacy (90). A sizable Swiss study discovered that ProADM was strong predictor of TF including death with an AUC that was significantly higher than that of CRP but similar to PCT (91-93). Similarly Copeptin has been shown to be a useful marker of early mortality or ICU admission, more so than other biomarkers (89). A small study of 51 patients demonstrated that copeptin was a significant predictor of ICU admission or mortality within 7 days, however PCT and MR proADM were not significant predictors of early decline (94). In a larger study of almost 370 patients copeptin was a more significant predictor of mortality than PCT, CRP and leukocyte count (89). Similarly in a large study from the German CAPNETZ group of 1740 copeptin was significantly higher in non survivors over 28 days more so than PCT and CRP (95).

Less favorable results surround the use of D Dimer, a product of fibrin degradation and a widely used, easily measured biomarker for thromboembolic disease. An elevated D Dimer level may be a non-specific finding in clinical practice (96). That said, D Dimer levels reflect the pathological role coagulation and fibrinolysis play in development acute lung injury. Levels increase in patients with severe CAP at high-risk mortality (97). For example, in an analysis of a subset of 147 patients 
from one RCT, an elevated D Dimer was associated with severe CAP, early TF and 30 days mortality. Levels were not significant for late TF (98). However, D Dimer does not appear improve the predictive scores such as the PSI and CURB $65(97,98)$.

\section{Immunoscore}

In sepsis, there is a complex interplay between host and pathogen in disease evolution. As highlighted by revised sepsis guidelines an important prognostic component of sepsis is the scale of inflammatory response, a factor that is neglected in existing pneumonia scores $(26,99)$. Therefor various studies have focused on enhancing the predictive ability of predictive scores through the addition of biomarkers $(24,58)$.

Studies focusing on integrating CRP with existing clinical predication scores provide a new intriguing insight in to this time-honored biomarker $(24,99)$. In a well-designed prospective study of 453 patients with CAP; the accuracy of predicting 30-day mortality through the use CRP, PCT and cytokines independently and in combination with the PSI and CURB65 scales was considered. The AUC of these prediction scales significantly increased following the addition of CRP (CURB-65 AUC increased from 0.82 to 0.85 and PSI AUC increased from 0.8 to 0.85 ). Interestingly the additive role of PCT to the clinical prediction scores in this study did not reach clinical significance (58). In another study by Menendez et al., the addition of CRP to the modified Halms score, improved the AUC to predict the absence of severe complications after day 3 from 0.77 to 0.84 $(\mathrm{P}=0.059)$ almost reaching significance. This increase in AUC was not reproduced on addition of PCT to the Halm score (25). The accuracy of the combined CRP-Halms score appears to improve with more complicated and severe disease $(24,25)$.

As with CRP, PCT is a promising adjunct to existing clinical prediction scores, and has a high negative predictive value for mortality when used in this way. In large multicentre study of patients with CAP diagnosed in the Emergency Department $(n=1,651)$, patient's with a PCT level of $<0.1 \mathrm{ng} / \mathrm{mL}$ had a significantly lower 30-day mortality rate, even those high clinical risk score as defined by the PSI and CURB 65. Therefor adding PCT improved the score's predictive ability (53). Similarly the CAPNETZ study group demonstrated that the combined use of CURB65 and PCT improved the prognostic accuracy compared with CURB-65 alone (AUC 0.83 vs. $0.79, \mathrm{P}<0.01$ ). The additional use of PCT using a threshold of $<0.228 \mathrm{ng} / \mathrm{mL}$ was able to predict patients with a low risk of death within all CURB-65 groups (100). Similar results have been demonstrated in other studies, however in two studies by Menendez et al. CRP was superior to PCT at predicting severe complications and mortality when added to selected severity scores $(25,58)$.

There were comparable results more novel biomarkers. The addition of IL-6 to existing clinical prediction scores improves accuracy. Zobel et al. demonstrated that including IL-6 in to a statistical model enhanced the CRB score with an AUC 0.8 compared to AUC of 0.701. In this study there was more modest increase in the AUC of CRB-65 with addition of IL-6 (29). Pro-ADM and copeptin have been shown to improve the prognostic accuracy of existing clinical prediction scores e.g., CURB 65 and PSI $(90,91)$. For example, in one study the combined use of PSI and Copeptin improves the AUC than for PSI alone (0.75 to 0.83) (94). Christ-Crain et al. similarly demonstrated that the addition of proADM to PSI increased the AUC from 0.73 to 0.77 (91).

\section{Conclusions}

In severe CAP the inflammatory response is complex and poorly controlled (101). As the interrogation of various immune-modulatory therapies intensify, so will the search for an appropriate surrogate of severity and treatment response (102). It is essential to deliver early, targeted treatment, and to continually assess the patient for an appropriate clinical response. It is important to be aware of the advantages and limitations of all available clinical tools. This review provides a concise summary of this rapidly evolving area. Diagnostic and predictive utility is likely to improve with a combination of scores and biomarkers, or even a biomarker panel, particularly in severely ill patients (99). Going forward it is essential to interrogate the evidence by rigorously analyzing data from large multisite trials, in a diverse cohort of patients.

\section{Acknowledgements}

None.

\section{Footnote}

Conflicts of Interest: The authors have no conflicts of interest to declare. 


\section{References}

1. Wunderink RG, Waterer GW. Community-acquired pneumonia. N Engl J Med 2014;370:1863.

2. Fine MJ, Smith MA, Carson CA, et al. Prognosis and outcomes of patients with community-acquired pneumonia. A meta-analysis. JAMA 1996;275:134-41.

3. Rello J, Rodriguez R, Jubert P, et al. Severe communityacquired pneumonia in the elderly: epidemiology and prognosis. Study Group for Severe Community-Acquired Pneumonia. Clin Infect Dis 1996;23:723-8.

4. Mandell LA, Wunderink RG, Anzueto A, et al. Infectious Diseases Society of America/American Thoracic Society consensus guidelines on the management of communityacquired pneumonia in adults. Clin Infect Dis 2007;44 Suppl 2:S27-72.

5. Roson B, Carratala J, Dorca J, et al. Etiology, reasons for hospitalization, risk classes, and outcomes of communityacquired pneumonia in patients hospitalized on the basis of conventional admission criteria. Clin Infect Dis 2001;33:158-65.

6. Dharmarajan K, Hsieh AF, Lin Z, et al. Diagnoses and timing of 30-day readmissions after hospitalization for heart failure, acute myocardial infarction, or pneumonia. JAMA 2013;309:355-63.

7. Takada K, Matsumoto S, Kojima E, et al. Predictors and impact of time to clinical stability in community-acquired pneumococcal pneumonia. Respir Med 2014;108:806-12.

8. Menendez R, Torres A, Zalacain R, et al. Risk factors of treatment failure in community acquired pneumonia: implications for disease outcome. Thorax 2004;59:960-5.

9. Menendez R, Torres A. Treatment failure in communityacquired pneumonia. Chest 2007;132:1348-55.

10. Torres A, Sibila O, Ferrer M, et al. Effect of corticosteroids on treatment failure among hospitalized patients with severe community-acquired pneumonia and high inflammatory response: a randomized clinical trial. JAMA 2015;313:677-86.

11. Menendez R, Torres A, Rodriguez de Castro F, et al. Reaching stability in community-acquired pneumonia: the effects of the severity of disease, treatment, and the characteristics of patients. Clin Infect Dis 2004;39:1783-90.

12. Genne D, Kaiser L, Kinge TN, et al. Community-acquired pneumonia: causes of treatment failure in patients enrolled in clinical trials. Clin Microbiol Infect 2003;9:949-54.

13. Halm EA, Fine MJ, Marrie TJ, et al. Time to clinical stability in patients hospitalized with community-acquired pneumonia: implications for practice guidelines. JAMA 1998;279:1452-7.

14. Aliberti S, Zanaboni AM, Wiemken T, et al. Criteria for clinical stability in hospitalised patients with communityacquired pneumonia. Eur Respir J 2013;42:742-9.

15. Hougham GW, Ham SA, Ruhnke GW, et al. Sequence patterns in the resolution of clinical instabilities in community-acquired pneumonia and association with outcomes. J Gen Intern Med 2014;29:563-71.

16. Finch S, Chalmers JD. Brief Clinical Review: Non Responding Pneumonia. EMJ Respir 2014;2:104-11.

17. Garcia-Vidal C, Carratala J. Early and late treatment failure in community-acquired pneumonia. Semin Respir Crit Care Med 2009;30:154-60.

18. Kolditz M, Ewig S, Hoffken G. Management-based risk prediction in community-acquired pneumonia by scores and biomarkers. Eur Respir J 2013;41:974-84.

19. Marti C, Garin N, Grosgurin O, et al. Prediction of severe community-acquired pneumonia: a systematic review and meta-analysis. Crit Care 2012;16:R141.

20. Aujesky D, Fine MJ. The pneumonia severity index: a decade after the initial derivation and validation. Clin Infect Dis 2008;47 Suppl 3:S133-9.

21. Schuetz P, Koller M, Christ-Crain M, et al. Predicting mortality with pneumonia severity scores: importance of model recalibration to local settings. Epidemiol Infect 2008;136:1628-37.

22. Kolditz M, Ewig S, Klapdor B, et al. Community-acquired pneumonia as medical emergency: predictors of early deterioration. Thorax 2015;70:551-8.

23. Guo Q, Li HY, Zhou YP, et al. CURB-65 score predicted mortality in community-acquired pneumonia better than IDSA/ATS minor criteria in a low-mortality-rate setting. Eur J Clin Microbiol Infect Dis 2012;31:3281-6.

24. Akram AR, Chalmers JD, Taylor JK, et al. An evaluation of clinical stability criteria to predict hospital course in community-acquired pneumonia. Clin Microbiol Infect 2013;19:1174-80.

25. Menendez R, Martinez R, Reyes S, et al. Stability in community-acquired pneumonia: one step forward with markers? Thorax 2009;64:987-92.

26. Singer M, Deutschman CS, Seymour CW, et al. The Third International Consensus Definitions for Sepsis and Septic Shock (Sepsis-3). JAMA 2016;315:801-10.

27. Povoa P. C-reactive protein: a valuable marker of sepsis. Intensive Care Med 2002;28:235-43.

28. Povoa P, Teixeira-Pinto AM, Carneiro AH. C-reactive protein, an early marker of community-acquired sepsis 
resolution: a multi-center prospective observational study. Crit Care 2011;15:R169.

29. Zobel K, Martus P, Pletz MW, et al. Interleukin 6, lipopolysaccharide-binding protein and interleukin 10 in the prediction of risk and etiologic patterns in patients with community-acquired pneumonia: results from the German competence network CAPNETZ. BMC Pulm Med 2012;12:6.

30. Andrijevic I, Matijasevic J, Andrijevic L, et al. Interleukin-6 and procalcitonin as biomarkers in mortality prediction of hospitalized patients with community acquired pneumonia. Ann Thorac Med 2014;9:162-7.

31. Prucha M, Bellingan G, Zazula R. Sepsis biomarkers. Clin Chim Acta 2015;440:97-103.

32. Gabay C, Kushner I. Acute-phase proteins and other systemic responses to inflammation. N Engl J Med 1999;340:448-54.

33. Moya F, Nieto A, JL RC. Calcitonin biosynthesis: evidence for a precursor. Eur J Biochem 1975;55:407-13.

34. Simon L, Gauvin F, Amre DK, et al. Serum procalcitonin and C-reactive protein levels as markers of bacterial infection: a systematic review and meta-analysis. Clin Infect Dis 2004;39:206-17.

35. Muller B, Harbarth S, Stolz D, et al. Diagnostic and prognostic accuracy of clinical and laboratory parameters in community-acquired pneumonia. BMC Infect Dis 2007;7:10.

36. McLean AS, Tang B, Huang SJ. Investigating sepsis with biomarkers. BMJ 2015;350:h254.

37. Heinrich PC, Castell JV, Andus T. Interleukin-6 and the acute phase response. Biochem J 1990;265:621-36.

38. Meisner M. Update on procalcitonin measurements. Ann Lab Med 2014;34:263-73.

39. Chalmers JD, Singanayagam A, Hill AT. C-reactive protein is an independent predictor of severity in communityacquired pneumonia. Am J Med 2008;121:219-25.

40. Almirall J, Bolibar I, Toran P, et al. Contribution of C-reactive protein to the diagnosis and assessment of severity of community-acquired pneumonia. Chest 2004;125:1335-42.

41. Sankar V, Webster NR. Clinical application of sepsis biomarkers. J Anesth 2013;27:269-83.

42. Kruger S, Ewig S, Kunde J, et al. Assessment of inflammatory markers in patients with communityacquired pneumonia--influence of antimicrobial pretreatment: results from the German competence network CAPNETZ. Clin Chim Acta 2010;411:1929-34.

43. Guervilly C, Coisel Y, Botelho-Nevers E, et al.
Significance of high levels of procalcitonin in patients with influenza A (H1N1) pneumonia. J Infect 2010;61:355-8.

44. Corsonello A, Luciani F, Incalzi RA. Biomarkers in community-acquired pneumonia: does chronic kidney disease matter? Am J Respir Crit Care Med 2011;183:1734; author response -5 .

45. Tilg H, Wilmer A, Vogel W, et al. Serum levels of cytokines in chronic liver diseases. Gastroenterology 1992;103:264-74.

46. Gilbert DN. Procalcitonin as a biomarker in respiratory tract infection. Clin Infect Dis 2011;52 Suppl 4:S346-50.

47. Rodriguez AH, Aviles-Jurado FX, Diaz E, et al. Procalcitonin (PCT) levels for ruling-out bacterial coinfection in ICU patients with influenza: A CHAID decision-tree analysis. J Infect 2016;72:143-51.

48. Sankar V, Webster NR. Clinical application of sepsis biomarkers. J Anesth 2013;27:269-83.

49. Silvestre JP, Coelho LM, Povoa PM. Impact of fulminant hepatic failure in C-reactive protein? J Crit Care 2010;25:657.e7-12.

50. El-Solh AA, Vora H, Knight PR 3rd, et al. Diagnostic use of serum procalcitonin levels in pulmonary aspiration syndromes. Crit Care Med 2011;39:1251-6.

51. Christ-Crain M, Muller B. Procalcitonin in bacterial infections--hype, hope, more or less? Swiss Med Wkly 2005;135:451-60.

52. Tang BM, Eslick GD, Craig JC, et al. Accuracy of procalcitonin for sepsis diagnosis in critically ill patients: systematic review and meta-analysis. Lancet Infect Dis 2007;7:210-7.

53. Huang DT, Weissfeld LA, Kellum JA, et al. Risk prediction with procalcitonin and clinical rules in communityacquired pneumonia. Ann Emerg Med 2008;52:48-58.e2.

54. Simpson RJ, Hammacher A, Smith DK, et al. Interleukin-6: structure-function relationships. Protein Sci 1997;6:929-55.

55. Hack CE, De Groot ER, Felt-Bersma RJ, et al. Increased plasma levels of interleukin-6 in sepsis. Blood 1989;74:1704-10.

56. Nishimoto N, Terao K, Mima T, et al. Mechanisms and pathologic significances in increase in serum interleukin-6 (IL-6) and soluble IL-6 receptor after administration of an anti-IL-6 receptor antibody, tocilizumab, in patients with rheumatoid arthritis and Castleman disease. Blood 2008;112:3959-64.

57. Kishimoto T. IL-6: from its discovery to clinical applications. Int Immunol 2010;22:347-52.

58. Menendez R, Martinez R, Reyes S, et al. Biomarkers 
improve mortality prediction by prognostic scales in community-acquired pneumonia. Thorax 2009;64:587-91.

59. Menendez R, Cavalcanti M, Reyes S, et al. Markers of treatment failure in hospitalised community acquired pneumonia. Thorax 2008;63:447-52.

60. Suresh MV, Singh SK, Ferguson DA, Jr., et al. Human C-reactive protein protects mice from Streptococcus pneumoniae infection without binding to pneumococcal C-polysaccharide. J Immunol 2007;178:1158-63.

61. Que YA, Virgini V, Lozeron ED, et al. Low C-reactive protein values at admission predict mortality in patients with severe community-acquired pneumonia caused by Streptococcus pneumoniae that require intensive care management. Infection 2015;43:193-9.

62. Bruns AH, Oosterheert JJ, Hak E, et al. Usefulness of consecutive C-reactive protein measurements in follow-up of severe community-acquired pneumonia. Eur Respir J 2008;32:726-32.

63. Coelho LM, Salluh JI, Soares M, et al. Patterns of c-reactive protein RATIO response in severe communityacquired pneumonia: a cohort study. Crit Care 2012;16:R53.

64. Boussekey N, Leroy O, Alfandari S, et al. Procalcitonin kinetics in the prognosis of severe community-acquired pneumonia. Intensive Care Med 2006;32:469-72.

65. Charles PE, Tinel C, Barbar S, et al. Procalcitonin kinetics within the first days of sepsis: relationship with the appropriateness of antibiotic therapy and the outcome. Crit Care 2009; 13:R38.

66. Martin-Loeches I, Valles X, Menendez R, et al. Predicting treatment failure in patients with community acquired pneumonia: a case-control study. Respir Res 2014;15:75.

67. Jensen JU, Heslet L, Jensen TH, et al. Procalcitonin increase in early identification of critically ill patients at high risk of mortality. Crit Care Med 2006;34:2596-602.

68. Stolz D, Christ-Crain M, Bingisser R, et al. Antibiotic treatment of exacerbations of COPD: a randomized, controlled trial comparing procalcitonin-guidance with standard therapy. Chest 2007;131:9-19.

69. Tang J, Long W, Yan L, et al. Procalcitonin guided antibiotic therapy of acute exacerbations of asthma: a randomized controlled trial. BMC Infect Dis 2013;13:596.

70. Albrich WC, Dusemund F, Bucher B, et al. Effectiveness and safety of procalcitonin-guided antibiotic therapy in lower respiratory tract infections in "real life": an international, multicenter poststudy survey (ProREAL). Arch Intern Med 2012;172:715-22.

71. Christ-Crain M, Stolz D, Bingisser R, et al. Procalcitonin guidance of antibiotic therapy in community-acquired pneumonia: a randomized trial. Am J Respir Crit Care Med 2006;174:84-93.

72. Schuetz P, Christ-Crain M, Thomann R, et al. Effect of procalcitonin-based guidelines vs standard guidelines on antibiotic use in lower respiratory tract infections: the ProHOSP randomized controlled trial. JAMA 2009;302:1059-66.

73. Christ-Crain M, Jaccard-Stolz D, Bingisser R, et al. Effect of procalcitonin-guided treatment on antibiotic use and outcome in lower respiratory tract infections: clusterrandomised, single-blinded intervention trial. Lancet 2004;363:600-7.

74. de Jong E, van Oers JA, Beishuizen A, et al. Efficacy and safety of procalcitonin guidance in reducing the duration of antibiotic treatment in critically ill patients: a randomised, controlled, open-label trial. Lancet Infect Dis 2016;16:819-27.

75. Bouadma L, Luyt CE, Tubach F, et al. Use of procalcitonin to reduce patients' exposure to antibiotics in intensive care units (PRORATA trial): a multicentre randomised controlled trial. Lancet 2010;375:463-74.

76. Schuetz P, Chiappa V, Briel M, et al. Procalcitonin algorithms for antibiotic therapy decisions: a systematic review of randomized controlled trials and recommendations for clinical algorithms. Arch Intern Med 2011;171:1322-31.

77. Ramirez P, Ferrer M, Marti V, et al. Inflammatory biomarkers and prediction for intensive care unit admission in severe community-acquired pneumonia. Crit Care Med 2011;39:2211-7.

78. Walley KR. Biomarkers in sepsis. Curr Infect Dis Rep 2013;15:413-20.

79. Povoa P, Salluh JI. Use of biomarkers in sepsis: many questions, few answers. Rev Bras Ter Intensiva 2013;25:1-2.

80. Seligman R, Papassotiriou J, Morgenthaler NG, et al. Copeptin, a novel prognostic biomarker in ventilatorassociated pneumonia. Crit Care 2008;12:R11.

81. Schluter B, Raufhake C, Erren M, et al. Effect of the interleukin-6 promoter polymorphism (-174 G/C) on the incidence and outcome of sepsis. Crit Care Med 2002;30:32-7.

82. Gallagher PM, Lowe G, Fitzgerald T, et al. Association of IL-10 polymorphism with severity of illness in community acquired pneumonia. Thorax 2003;58:154-6.

83. Martin-Loeches I, Sole-Violan J, Rodriguez de Castro F, et al. Variants at the promoter of the interleukin- 6 gene 
are associated with severity and outcome of pneumococcal community-acquired pneumonia. Intensive Care Med 2012;38:256-62.

84. Struck J, Tao C, Morgenthaler NG, et al. Identification of an Adrenomedullin precursor fragment in plasma of sepsis patients. Peptides 2004;25:1369-72.

85. Wang P. Adrenomedullin in sepsis and septic shock. Shock 1998;10:383-4.

86. Martinez A, Pio R, Zipfel PF, et al. Mapping of the adrenomedullin-binding domains in human complement factor H. Hypertens Res 2003;26 Suppl:S55-9.

87. Morgenthaler NG, Muller B, Struck J, et al. Copeptin, a stable peptide of the arginine vasopressin precursor, is elevated in hemorrhagic and septic shock. Shock 2007;28:219-26.

88. Katan M, Muller B, Christ-Crain M. Copeptin: a new and promising diagnostic and prognostic marker. Crit Care 2008; 12:117.

89. Muller B, Morgenthaler N, Stolz D, et al. Circulating levels of copeptin, a novel biomarker, in lower respiratory tract infections. Eur J Clin Invest 2007;37:145-52.

90. Cavallazzi R, El-Kersh K, Abu-Atherah E, et al. Midregional proadrenomedullin for prognosis in community-acquired pneumonia: a systematic review. Respir Med 2014;108:1569-80.

91. Christ-Crain M, Morgenthaler NG, Stolz D, et al. Proadrenomedullin to predict severity and outcome in community-acquired pneumonia [ISRCTN04176397]. Crit Care 2006;10:R96.

92. Gordo-Remartinez S, Calderon-Moreno M, Fernandez-Herranz J, et al. Usefulness of midregional proadrenomedullin to predict poor outcome in patients with community acquired pneumonia. PLoS One 2015;10:e0125212.

93. Claessens YE, Mathevon T, Kierzek G, et al. Accuracy of C-reactive protein, procalcitonin, and mid-regional pro-atrial natriuretic peptide to guide site of care of

Cite this article as: Morley D, Torres A, Cillóniz C, MartinLoeches I. Predictors of treatment failure and clinical stability in patients with community acquired pneumonia. Ann Transl Med 2017;5(22):443. doi: 10.21037/atm.2017.06.54 community-acquired pneumonia. Intensive Care Med 2010;36:799-809.

94. Kolditz M, Halank M, Schulte-Hubbert B, et al. Copeptin predicts clinical deterioration and persistent instability in community-acquired pneumonia. Respir Med 2012;106:1320-8.

95. Kruger S, Ewig S, Kunde J, et al. Pro-atrial natriuretic peptide and pro-vasopressin for predicting short-term and long-term survival in community-acquired pneumonia: results from the German Competence Network CAPNETZ. Thorax 2010;65:208-14.

96. Kabrhel C, Mark Courtney D, Camargo CA Jr, et al. Factors associated with positive D-dimer results in patients evaluated for pulmonary embolism. Acad Emerg Med 2010;17:589-97.

97. Querol-Ribelles JM, Tenias JM, Grau E, et al. Plasma d-dimer levels correlate with outcomes in patients with community-acquired pneumonia. Chest 2004;126:1087-92.

98. Snijders D, Schoorl M, Schoorl M, et al. D-dimer levels in assessing severity and clinical outcome in patients with community-acquired pneumonia. A secondary analysis of a randomised clinical trial. Eur J Intern Med 2012;23:436-41.

99. Yang Y, Xie J, Guo F, et al. Combination of C-reactive protein, procalcitonin and sepsis-related organ failure score for the diagnosis of sepsis in critical patients. Ann Intensive Care 2016;6:51.

100. Kruger S, Ewig S, Marre R, et al. Procalcitonin predicts patients at low risk of death from community-acquired pneumonia across all CRB-65 classes. Eur Respir J 2008;31:349-55.

101. Antunes G, Evans SA, Lordan JL, et al. Systemic cytokine levels in community-acquired pneumonia and their association with disease severity. Eur Respir J 2002;20:990-5.

102. Kox WJ, Volk T, Kox SN, et al. Immunomodulatory therapies in sepsis. Intensive Care Med 2000;26 Suppl 1:S124-8. 